\title{
General pairing theory for condensed and non-condensed pairs of a superconductor in a high magnetic field
}

\author{
Peter Scherpelz, ${ }^{1}$ Dan Wulin, ${ }^{1}$ Břetislav Šopík, ${ }^{1}$ K. Levin, ${ }^{1}$ and A. K. Rajagopal ${ }^{2,3}$ \\ ${ }^{1}$ James Franck Institute and Department of Physics, \\ University of Chicago, Chicago, Illinois 60637, USA \\ ${ }^{2}$ Inspire Institute Inc., Alexandria, Virginia 22303, USA \\ ${ }^{3}$ Harish-Chandra Research Institute, Chhatnag Road, Jhunsi, Allahabad, 211019, India
}

(Dated: May 24, 2022)

\begin{abstract}
We extend Gor'kov theory to address superconducting pairing at high magnetic fields and general temperatures with arbitrary attractive interaction strength. This analysis begins with a new interpretation of the high-field Gor'kov gap equation which we associate with an instability in a generalized particle-particle ladder series. Importantly, this interpretation of the non-linear gap equation enables a treatment of pairing which is distinct from condensation. We also show how to consolidate two distinct fermionic pairing schemes in real and momentum space, both corresponding to an Abrikosov lattice. Numerical results for the fermionic local density of states demonstrate that gapless structure in a field is robust and presumably relevant to quantum oscillation experiments. We find that despite their differences, both pairing schemes contain very similar physics. Our formalism is designed to explore a variety of magnetic field effects in the so-called pseudogap phase and throughout the BCS-BEC crossover.
\end{abstract}

\section{INTRODUCTION}

High magnetic field superconductivity is a difficult theoretical problem with important implications for experiment. Observations of quantum oscillations in the superconducting phase of conventional superconductors were initially unexpected, ${ }^{1]}$ and further unusual oscillations have been observed in underdoped cuprates ${ }^{2]}$ At the same time, theoretical treatments reached surprising conclusions, with some investigations finding re-entrant superconductivity at extremely high magnetic fields. ${ }^{3}$ Adding to the complexity is the observation that the introduction of Landau levels into a pairing scheme appears to cause a three-dimensional superconductor to behave like a one-dimensional system. This greatly enhances fluctuation effects ${ }^{4}$ and may even de-stabilize superfluid condensation.

The goal of the present paper is to set up a foundation for addressing these issues by extending the standard Gor'kov (BCS-based) approach to apply to general temperatures $T$, away from the instability regime, and to stronger-than-BCS attractive interactions. Although the immediate focus of this paper is on the ordered phase, we use the Gor'kov theory extension to arrive at a compatible description of the normal phase as well. Importantly, this normal phase may possess a rich structure associated with precursor pairing in the presence of magnetic fields.

In a related paper ${ }^{5}$ we focus on this disordered phase and explore the notion that some degree of pairing at the onset of condensation may be necessary to avoid a strict dimensional reduction that prohibits condensation into a superconducting state altogether. Furthermore, these excited pair states may be present in systems such as high temperature superconductors or in fermionic gases in the BCS-BEC crossover regime, where the non-condensed pairs are associated with a pseudogap state. It should be stressed that the non-condensed pairs we consider are distinct from conventional fluctuations. These pairs arise from strong attractive interactions, not from low dimensionality (and/or disorder effects) which give rise to conventional fluctuations. Our BCS-BEC based approach is similarly distinct from the so-called "phase fluctuation" scenario which is based on soft phase fluctuations presumably arising from low plasma frequencies. Indeed, since we are contemplating both charged and uncharged superfluids, the issue of soft plasma frequencies is not particularly generic.

Gor'kov theory addresses the fermionic degrees of freedom. Two proposals $\sqrt{617}$ have been put forth to describe the nature of those fermionic pairs which form the condensate in the presence of high magnetic fields. These are associated with orbit center-based and magnetic latticebased pairing schemes. Here we show how the physical implications of each are similar and that both lead to gapless fermionic states which are thought to be the basis for observed quantum oscillations. ${ }^{1} \mathrm{~A}$ central contribution of the present paper is to demonstrate that the (analytically tractable) theoretical structure of these different pairing approaches can be consolidated into a more general formulation which addresses the non-linear structure of the Gor'kov theory. This is in contrast to a substantial fraction of the literature on high magnetic field superconductivity which deals with the linear regime where the gap is small. We thus arrive at an interpretation of the Gor'kov gap equation which allows us to extract a set of particle-particle ladder diagrams which properly characterize the pairing fluctuations or non-condensed pairs in the presence of high magnetic fields.

We begin in Section II by deriving the Landau level representation of the Gor'kov equations. In Section III we discuss a "diagonal" approximation made to these equations which is thought ${ }^{8}$ to be suitable for high magnetic fields. Following this, in section [IV] we focus on the 
gap equation and discuss its relationship to a divergent particle-particle ladder series. In section $\mathrm{V}$ we show that this divergent series captures the contribution of noncondensed pairs to the theory.

The second half of the paper is less general and more concrete. Here we focus on implementing and comparing different state-space representations of this pairing theory. In Section VI we discuss the two different existing implementations. In Section VII we show how to use a "tight-binding" approximation to orbit-center pairing to make the theory analytically tractable, facilitating comparison of the two implementations. Finally, in Section VIII we show how gaplessness is robust among these pairing theories by comparing local density of states calculations. Our conclusions are presented in Section IX.

\section{DERIVATION OF GOR'KOV'S EQUATIONS IN A LANDAU LEVEL BASIS}

We begin with the Gor'kov equations formulated in real space in terms of the gap $\Delta(\boldsymbol{r})$ and Green's functions in integral form:

$$
\begin{gathered}
G\left(\boldsymbol{r}, \boldsymbol{r}^{\prime} ; i \omega\right)=G_{0}\left(\boldsymbol{r}, \boldsymbol{r}^{\prime} ; i \omega\right)-\int d \boldsymbol{r}^{\prime \prime} d \boldsymbol{r}^{\prime \prime \prime} G_{0}\left(\boldsymbol{r}, \boldsymbol{r}^{\prime \prime} ; i \omega\right) \\
\times \Delta\left(\boldsymbol{r}^{\prime \prime}\right) G_{0}\left(\boldsymbol{r}^{\prime \prime \prime}, \boldsymbol{r}^{\prime \prime} ;-i \omega\right) \Delta^{\dagger}\left(\boldsymbol{r}^{\prime \prime \prime}\right) G\left(\boldsymbol{r}^{\prime \prime \prime}, \boldsymbol{r}^{\prime} ; i \omega\right) \\
\Delta^{\dagger}(\boldsymbol{r})=-\frac{g}{\beta} \sum_{i \omega} \int d \boldsymbol{r}^{\prime} G\left(\boldsymbol{r}^{\prime}, \boldsymbol{r} ; i \omega\right) G_{0}\left(\boldsymbol{r}^{\prime}, \boldsymbol{r} ;-i \omega\right) \Delta^{\dagger}\left(\boldsymbol{r}^{\prime}\right)
\end{gathered}
$$

where $g$ is the interaction strength, and $i \omega(i \Omega)$ denote fermionic (bosonic) Matsubara frequencies with the traditional subscripts omitted for clarity. These equations allow the identification of the self-energy $\Sigma$,

$$
\Sigma\left(\boldsymbol{r}, \boldsymbol{r}^{\prime} ; i \omega\right)=-\Delta(\boldsymbol{r}) \Delta^{\dagger}\left(\boldsymbol{r}^{\prime}\right) G_{0}\left(\boldsymbol{r}^{\prime}, \boldsymbol{r} ;-i \omega\right),
$$

and we now rewrite Eqs. (1-3) in terms of the Landau level representation that diagonalizes the non-interacting Hamiltonian $\mathcal{H}_{0} \cdot 9$ The bare Green's function $G_{0}$ is then given by

$$
\begin{aligned}
G_{0}\left(\boldsymbol{r}, \boldsymbol{r}^{\prime} ; i \omega\right) & =\sum_{n} G_{n}^{0}(i \omega) \psi_{n}(\boldsymbol{r}) \psi_{n}^{\dagger}\left(\boldsymbol{r}^{\prime}\right) \\
& =\sum_{n} \frac{\psi_{n}(\boldsymbol{r}) \psi_{n}^{\dagger}\left(\boldsymbol{r}^{\prime}\right)}{i \omega-\xi_{n}}
\end{aligned}
$$

where $n=\left(N, p, k_{z}\right)$ is the Landau level state, with $N$ the Landau level, $p$ the degenerate Landau level index, $k_{z}$ the momentum in the $z$-direction (parallel to a magnetic field $\boldsymbol{B}$ ), and $\xi_{n}$ the energy of a particle in state $n$. $G$, however, is not in general diagonal in the Landau level representation, and is given by

$$
G\left(\boldsymbol{r}, \boldsymbol{r}^{\prime} ; i \omega\right)=\sum_{m m^{\prime}} G_{m m^{\prime}}(i \omega) \psi_{m}(\boldsymbol{r}) \psi_{m^{\prime}}^{\dagger}\left(\boldsymbol{r}^{\prime}\right)
$$

In this representation, Eq. (1) is multiplied by $\psi_{m}^{\dagger}(\boldsymbol{r}) \psi_{m^{\prime}}\left(\boldsymbol{r}^{\prime}\right)$ and integrated over both $\boldsymbol{r}$ and $\boldsymbol{r}^{\prime}$ to give

$$
\begin{aligned}
G_{m m^{\prime}} & (i \omega)=G_{m}^{0}(i \omega) \delta_{m m^{\prime}}-\sum_{l n} \int d \boldsymbol{r}^{\prime \prime} d \boldsymbol{r}^{\prime \prime \prime} G_{m}^{0}(i \omega) \\
& \times \psi_{m}^{\dagger}\left(\boldsymbol{r}^{\prime \prime}\right) \Delta\left(\boldsymbol{r}^{\prime \prime}\right) G_{l}^{0}(-i \omega) \psi_{l}\left(\boldsymbol{r}^{\prime \prime \prime}\right) \psi_{l}^{\dagger}\left(\boldsymbol{r}^{\prime \prime}\right) \Delta^{\dagger}\left(\boldsymbol{r}^{\prime \prime \prime}\right) \\
& \times G_{n m^{\prime}}(i \omega) \psi_{n}\left(\boldsymbol{r}^{\prime \prime \prime}\right) .
\end{aligned}
$$

We then define a "state-space gap"

$$
\Delta_{m n} \equiv \int d \boldsymbol{r} \Delta(\boldsymbol{r}) \psi_{m}^{\dagger}(\boldsymbol{r}) \psi_{n}^{\dagger}(\boldsymbol{r})
$$

and obtain

$$
\begin{aligned}
G_{m m^{\prime}}(i \omega)= & G_{m}^{0}(i \omega) \delta_{m m^{\prime}}-\sum_{l n} G_{m}^{0}(i \omega) \Delta_{m l} \\
& \times G_{l}^{0}(-i \omega) \Delta_{l n}^{\dagger} G_{n m^{\prime}}(i \omega) .
\end{aligned}
$$

We multiply the gap equation, Eq. (2), by $\Delta(\boldsymbol{r})$, and express the right hand side in the Landau level representation to find

$$
\begin{aligned}
& \int d \boldsymbol{r}|\Delta(\boldsymbol{r})|^{2}=-\frac{g}{\beta} \sum_{m m^{\prime} n} \sum_{i \omega} \int d \boldsymbol{r}^{\prime} d \boldsymbol{r} G_{m m^{\prime}}(i \omega) \\
& \times G_{n}^{0}(-i \omega) \Delta^{\dagger}\left(\boldsymbol{r}^{\prime}\right) \Delta(\boldsymbol{r}) \psi_{m}\left(\boldsymbol{r}^{\prime}\right) \psi_{m^{\prime}}^{\dagger}(\boldsymbol{r}) \psi_{n}\left(\boldsymbol{r}^{\prime}\right) \psi_{n}^{\dagger}(\boldsymbol{r}) .
\end{aligned}
$$

Using the above identity for $\Delta_{m n}$, the gap equation becomes

$$
1=-\frac{g}{\beta} \sum_{m m^{\prime} n} \sum_{i \omega} \frac{\Delta_{m^{\prime} n} \Delta_{m n}^{\dagger}}{\int d \boldsymbol{r}|\Delta(\boldsymbol{r})|^{2}} G_{m m^{\prime}}(i \omega) G_{n}^{0}(-i \omega) .
$$

The self-energy is similarly expressed in the Landau level representation,

$$
\Sigma\left(\boldsymbol{r}, \boldsymbol{r}^{\prime} ; i \omega\right)=\sum_{m m^{\prime}} \Sigma_{m m^{\prime}}(i \omega) \psi_{m}(\boldsymbol{r}) \psi_{m^{\prime}}^{\dagger}\left(\boldsymbol{r}^{\prime}\right) .
$$

Using Eq. 3 for $\Sigma$, we find

$$
\Sigma_{m m^{\prime}}(i \omega)=-\sum_{n} G_{n}^{0}(-i \omega) \Delta_{m n} \Delta_{n m^{\prime}}^{\dagger}
$$

In summary, the Gor'kov equations in the Landau level representation for a constant magnetic field are

$$
\begin{gathered}
G_{m m^{\prime}}(i \omega)=G_{m}^{0}(i \omega) \delta_{m m^{\prime}}-\sum_{l n} G_{m}^{0}(i \omega) \Delta_{m l} \\
\times G_{l}^{0}(-i \omega) \Delta_{l n}^{\dagger} G_{n m^{\prime}}(i \omega) \\
1=-\frac{g}{\beta} \sum_{m m^{\prime} n} \sum_{i \omega} \frac{\Delta_{m^{\prime} n} \Delta_{m n}^{\dagger}}{\int d \boldsymbol{r}|\Delta(\boldsymbol{r})|^{2}} G_{m m^{\prime}}(i \omega) G_{n}^{0}(-i \omega) \\
\Sigma_{m m^{\prime}}(i \omega)=-\sum_{n} G_{n}^{0}(-i \omega) \Delta_{m n} \Delta_{n m^{\prime}}^{\dagger}
\end{gathered}
$$




\section{INTRA-EIGENSTATE PAIRING}

To make further analytical progress, we must simplify

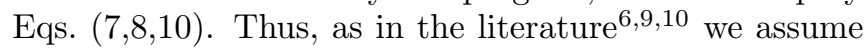
that the pairing involves degenerate eigenstates. This assumption is justified if we are in a regime where $|\Delta|$ is much less than the splitting between the Landau levels $\hbar \omega_{c}$, where $\omega_{c}=e H /(\hbar c m)$ is the cyclotron frequency. This will be a good approximation at very high fields where the splitting between Landau levels is large, the regime we explore in this paper. This approach has been analyzed carefully in Ref. 8 which showed that in this regime inter-Landau level effects that we neglect are perturbative.

We also simplify by defining

$$
\Delta_{m n}^{0}(\zeta) \equiv \frac{\Delta_{m n}(\zeta)}{\sqrt{\int d \boldsymbol{r}|\Delta(\boldsymbol{r}, \zeta)|^{2}}} \equiv \frac{\Delta_{m n}(\zeta)}{\Delta} .
$$

Note that we have introduced an important parameter $\zeta$ which labels different functional forms of $\Delta(\boldsymbol{r})$. Throughout this paper we will associate the particular value $\zeta_{0}$ with the condensate configuration $\Delta^{\mathrm{sc}}\left(\boldsymbol{r}, \zeta_{0}\right)$, which may be distinct from configurations occupied by non-condensed pairs, discussed below. It is convenient to define

$$
\phi_{m m^{\prime}}^{2}(\zeta) \equiv \sum_{n} \Delta_{m n}^{0}(\zeta) \Delta_{n m^{\prime}}^{0 \dagger}(\zeta)
$$

In order that all the potential pairing partners $n$ of a state $m$ are energy degenerate, the index $n$ is in the same Landau level as $m$, and pairing occurs between states with $z$-momenta $k_{z}$ and $-k_{z}$. This allows us to write the fermionic self-energy Eq. 10 as

$$
\Sigma_{m m^{\prime}}(i \omega)=-G_{N}^{0}\left(k_{z} ;-i \omega\right)|\Delta|^{2} \phi_{m m^{\prime}}^{2}\left(\zeta_{0}\right),
$$

where we write $G^{0}$ in terms of only the Landau level $N$ and the $z$-momentum $k_{z}$ of $m$, with $N_{m}=N_{m^{\prime}}$ and $k_{z_{m}}=k_{z_{m^{\prime}}}$. This simplification of the gap equation, Eq. (8), leads to

$$
1=-\frac{g}{\beta} \sum_{m m^{\prime}} \sum_{i \omega} \phi_{m m^{\prime}}^{2}\left(\zeta_{0}\right) G_{m m^{\prime}}(i \omega) G_{N}^{0}\left(k_{z} ;-i \omega\right) .
$$

\section{THE GAP EQUATION AS DIVERGENT PARTICLE-PARTICLE LADDER SERIES}

The above formulation enables a consolidation of standard approaches in the literature $6 / 7 / 9 / 10$ In this context we re-interpret the gap equation of Eq. (14) as a generalized Thouless condition, which applies to all temperatures below the transition. In the process we show that this gap equation serves to identify a particular particleparticle ladder series. The divergence of this series is a prerequisite for, and an indicator of, the superconducting state. Importantly this ladder series also leads us to a characterization of the associated non-condensed pairs, which may form above the critical temperature for stronger-than-BCS interactions.

It is essential first to characterize the degrees of freedom available to these non-condensed pairs. In the $z$ direction parallel to the magnetic field, the system behaves as in zero-field, where condensed pairs are constructed of fermions with momenta $k_{z}$ and $-k_{z}$. Thus, excited pairs must have nonzero total momentum, and we can describe the general pairing of non-condensed pairs as being between momenta $k_{z}$ and $-k_{z}+q_{z}$.11

In the plane perpendicular to the magnetic field, the condensed electrons are those which pair to form the realspace superconducting gap. Here and throughout we distinguish the order parameter $\Delta^{\mathrm{sc}}\left(\boldsymbol{r}, \zeta_{0}\right)$ from the excitation gap. In a mean-field scheme, such as ours, where individual vortex fluctuations are not included, we assume that $\zeta_{0}$ corresponds to an Abrikosov lattice with functional form $\Delta^{0}\left(\boldsymbol{r}, \zeta_{0}\right)=\Delta^{\mathrm{sc}}\left(\boldsymbol{r}, \zeta_{0}\right) / \Delta$. We take the non-condensed pairs to be those that form other realspace gaps $\Delta^{0}(\boldsymbol{r}, \zeta)$ for $\zeta \neq \zeta_{0}$. Finally, we also allow the Matsubara frequencies to appear with total frequency $i \Omega$.

We next introduce the pair susceptibility, $\chi\left(\zeta, q_{z} ; i \Omega\right) \equiv$

$$
\frac{1}{\beta} \sum_{m m^{\prime}} \sum_{i \omega} \phi_{m m^{\prime}}^{2}(\zeta) G_{m m^{\prime}}(i \omega) G_{N}^{0}\left(k_{z}-q_{z} ; i \Omega-i \omega\right)
$$

With this important definition, and the introduction of non-condensed pairs, we return to the gap equation, Eq. 14, which can be rewritten as

$$
1+g \chi\left(\zeta=\zeta_{0}, q_{z}=0 ; i \Omega=0\right)=0 .
$$

One can interpret this equation as reflecting a divergence of a particle-particle ladder summation, shown in Fig. 1. We argue below that the gap equation is to be associated with a $t$-matrix formed from the ladder diagrams in Fig. 1. given here in an abbreviated form by

$$
t^{\mathrm{pg}}\left(\zeta, q_{z} ; i \Omega\right)=\frac{g}{1+g \chi\left(\zeta, q_{z}, i \Omega\right)}
$$

(see Section $\mathrm{V}$ for details). For the condensate configuration, $\zeta=\zeta_{0}, q_{z}=\Omega=0$, the $t$-matrix thus diverges for all temperatures below the instability, as in a Bose Einstein condensation condition, where the pairs have vanishing chemical potential. This ladder diagram set is to be distinguished from a series which was previously identified to correspond to the specific instability point Ref. 12. Here, the condition is stronger, as the incorporation of one dressed $G$ and one bare $G^{0}$ makes this summation valid throughout the superconducting regime. Furthermore, as we explain in Section V it can be extended to the entire strong attraction (BCS-BEC) regime where there is a finite excitation gap at the instability, and in which pairing and condensation must be distinguished! $\lcm{511}$ 

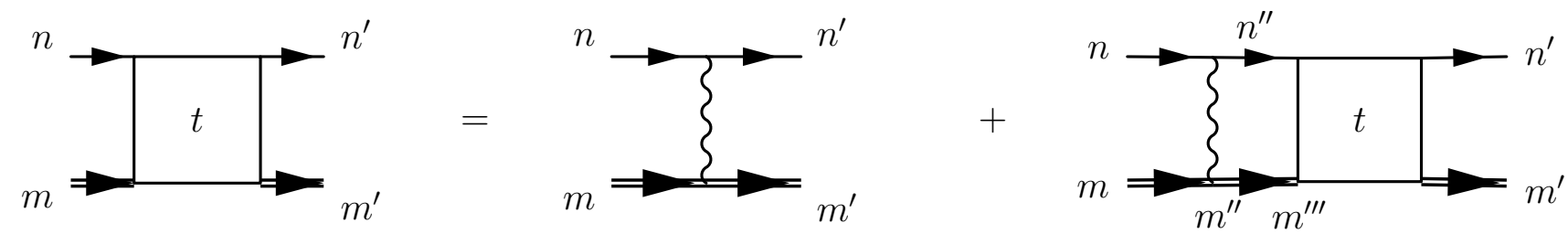

FIG. 1. The particle-particle ladder summation corresponding to a $t$-matrix $t^{\mathrm{pg}}$, the divergence of which gives the general gap equation for the superconducting state.

\section{CHARACTERIZING THE NON-CONDENSED PAIRS IN THE GOR'KOV EQUATIONS}

BCS theory represents a very special case of superfluidity in which pairing and condensation take place at the same temperature. We have just argued that the Gor'kov gap equation of Eq. (16) and the closely related $t$-marix of Eq. 17) effectively constrain the nature of non-condensed pairs which below the transition condense into a state with gap structure $\Delta^{0}\left(\boldsymbol{r}, \zeta_{0}\right)$.

For the moment, we consider Eq. (17) as an appropriate characterization of the $t$-matrix (or effective propagator) associated with the non-condensed pairs. We next characterize their feedback into the Gor'kov equations, by including $t$ in the self energy. In a strict Gor'kov theory $\Sigma_{m m^{\prime}}^{\mathrm{sc}}(i \omega)=$

$$
\sum_{\zeta, q_{z}, i \Omega} \phi_{m m^{\prime}}^{2}(\zeta) t_{m m^{\prime}}^{\mathrm{sc}}\left(\zeta, q_{z} ; i \Omega\right) G_{N}^{0}\left(q_{z}-k_{z} ; i \Omega-i \omega\right)
$$

where

$$
t^{\mathrm{sc}}\left(\zeta, q_{z} ; i \Omega\right) \equiv-\delta\left(\zeta-\zeta_{0}\right) \delta\left(q_{z}\right) \delta(i \Omega)\left|\Delta^{\mathrm{sc}}\right|^{2},
$$

with $\Delta^{\mathrm{sc}}$ corresponding to the gap $\Delta$ in Eq. (11).

We now include in the self-energy the non-condensed pair propagator $t^{p g}$ given by Eq. (17), and in this way go beyond strict Gor'kov theory. This contribution is $\Sigma_{m m^{\prime}}^{\mathrm{pg}}(i \omega)=$

$$
\sum_{\zeta, q_{z}, i \Omega} \phi_{m m^{\prime}}^{2}(\zeta) t^{\mathrm{pg}}\left(\zeta, q_{z} ; i \Omega\right) G_{N}^{0}\left(q_{z}-k_{z} ; i \Omega-i \omega\right) .
$$

Furthermore, since $1+g \chi\left(\zeta_{0}, 0 ; 0\right)$ diverges below the critical temperature, $t^{\mathrm{pg}}$ will be strongly peaked for parameters $\left(\zeta, q_{z} ; i \Omega\right) \approx\left(\zeta_{0}, 0 ; 0\right)$. Since $i \Omega$ and $q_{z}$ will be small for the primary contributions to $t^{\mathrm{pg}}$, we approximate the right hand side using $G_{N}^{0}\left(q_{z}-k_{z} ; i \Omega-i \omega\right) \approx$ $G_{N}^{0}\left(-k_{z} ;-i \omega\right)$.

Then the total self-energy is now

$$
\begin{aligned}
\Sigma_{m m^{\prime}} & (i \omega)=\Sigma_{m m^{\prime}}^{\mathrm{sc}}(i \omega)+\Sigma_{m m^{\prime}}^{\mathrm{pg}}(i \omega) \\
= & G_{N}^{0}\left(-k_{z} ;-i \omega\right) \sum_{\zeta, q_{z}, i \Omega} \phi_{m m^{\prime}}^{2}(\zeta) \\
& \times\left(t^{\mathrm{sc}}\left(\zeta, q_{z} ; i \Omega\right)+t^{\mathrm{pg}}\left(\zeta, q_{z} ; i \Omega\right)\right),
\end{aligned}
$$

where $\Sigma_{m m^{\prime}}^{\mathrm{sc}}$ is the original self-energy from Gor'kov theory defined in Eq. (10), and $\Sigma_{m m^{\prime}}^{\mathrm{pg}}$ is defined in Eq. 20.

We can further simplify by defining a total $|\Delta|^{2}$,

$$
|\Delta|^{2} \equiv\left|\Delta^{\mathrm{sc}}\right|^{2}+\left|\Delta^{\mathrm{pg}}\right|^{2}
$$

with

$$
\left|\Delta^{\mathrm{pg}}\right|^{2} \equiv-\sum_{\zeta, q_{z} ; i \Omega} \frac{g}{1+g \chi\left(\zeta, q_{z} ; i \Omega\right)}
$$

and $\left\langle\phi_{m m^{\prime}}^{2}(\zeta)\right\rangle_{\zeta} \equiv$

$$
\frac{\sum_{\zeta, q_{z}, i \Omega} \phi_{m m^{\prime}}^{2}(\zeta)\left(t^{\mathrm{sc}}\left(\zeta, q_{z} ; i \Omega\right)+t^{\mathrm{pg}}\left(\zeta, q_{z} ; i \Omega\right)\right)}{\sum_{\zeta, q_{z}, i \Omega}\left(t^{\mathrm{sc}}\left(\zeta, q_{z} ; i \Omega\right)+t^{\mathrm{pg}}\left(\zeta, q_{z} ; i \Omega\right)\right)} .
$$

This leads to an expression for the self-energy which can be written compactly as

$$
\Sigma_{m m^{\prime}}(i \omega)=-G_{N}^{0}\left(-k_{z} ;-i \omega\right)\left\langle\phi_{m m^{\prime}}^{2}(\zeta)\right\rangle_{\zeta}|\Delta|^{2} .
$$

Together with the number equation $N=$ $\frac{2}{\beta} \sum_{m, i \omega} G_{m m}(i \omega)$ and Eqs. 78 this forms a system of equations which can be solved self-consistently! 11 In this way we have modified the Gor'kov theory to extend BCS theory into the more general regime where pairing and condensation are treated differently.

Finally, we turn to a more detailed interpretation of the ladder diagrams and the related pair susceptibility. In Fig. 1, particles forming a pair may interact to create a new non-condensed pair in the same excited pair state. That is, we consider a pairing propagator $t_{m n}^{m^{\prime} n^{\prime}, \operatorname{pg}}\left(\zeta, q_{z} ; i \Omega\right)$ between states $m, n$ and $m^{\prime}, n^{\prime}$, where $m=\left(N, p, k_{z}, i \omega\right)$ and $n=\left(N^{\prime}, p^{\prime}, q_{z}-k_{z}, i \Omega-i \omega\right)$ are pairing partners for a given real-space configuration $\zeta$, total $z$-momentum $q_{z}$, and total Matsubara frequency $i \Omega$, and $m^{\prime}=\left(N^{\prime \prime}, p^{\prime \prime}, k_{z}^{\prime}, i \omega^{\prime}\right)$ and $n^{\prime}=\left(N^{\prime \prime \prime}, p^{\prime \prime \prime}, q_{z}-\right.$ $\left.k_{z}^{\prime}, i \Omega-i \omega^{\prime}\right)$ are pairing partners sharing the same $\zeta$, $q_{z}$ and $i \Omega$. Note that $m$ now includes frequency, and intra-eigenstate pairing is not enforced here. The bare interaction between the two pairs, as an extension of the BCS mean-field Hamiltonian, is in a separable form $g V_{m n}^{m^{\prime} n^{\prime}}=g \Delta_{m n}^{0 \dagger}(\zeta) \Delta_{m^{\prime} n^{\prime}}^{0}(\zeta)$. Then the infinite particleparticle ladder summation is

$$
\begin{aligned}
& t_{m n}^{m^{\prime} n^{\prime}, \mathrm{pg}}\left(\zeta, q_{z} ; i \Omega\right)=g V_{m n}^{m^{\prime} n^{\prime}}- \\
& g V_{m n}^{m^{\prime \prime} n^{\prime \prime}} G_{n^{\prime \prime}}^{0} G_{m^{\prime \prime} m^{\prime \prime \prime}} t_{m^{\prime \prime \prime} n^{\prime \prime}}^{m^{\prime} n^{\prime}, \mathrm{pg}}\left(\zeta, q_{z} ; i \Omega\right) .
\end{aligned}
$$


As $V$ is separable between $m, n$ and $m^{\prime}, n^{\prime}$, this infinite summation has the solution

$$
\begin{aligned}
& t_{m n}^{m^{\prime} n^{\prime}, \operatorname{pg}}\left(\zeta, q_{z} ; i \Omega\right)= \\
& \frac{g V_{m n}^{m^{\prime} n^{\prime}}}{1+g \sum_{m^{\prime \prime}, m^{\prime \prime \prime}, n^{\prime \prime}} V_{m^{\prime \prime \prime} n^{\prime \prime}}^{m^{\prime \prime} n^{\prime \prime}} G_{n^{\prime \prime}}^{0} G_{m^{\prime \prime} m^{\prime \prime \prime}}} .
\end{aligned}
$$

Using the expression for $V$, and applying the intraeigenstate pairing approximation, this further simplifies to

$$
\begin{aligned}
& t_{m n}^{m^{\prime} n^{\prime}, \mathrm{pg}}\left(\zeta, q_{z} ; i \Omega\right)= \\
& \frac{g \Delta_{m n}^{0 \dagger}(\zeta) \Delta_{m^{\prime} n^{\prime}}^{0}(\zeta)}{1+g \sum_{m^{\prime \prime}, m^{\prime \prime \prime}} \phi_{m^{\prime \prime} m^{\prime \prime \prime}}^{2}(\zeta) G_{m^{\prime \prime} m^{\prime \prime}} G_{N}^{0}\left(k_{z}-q_{z} ; i \Omega-i \omega\right)} .
\end{aligned}
$$

This in turn gives the $t$-matrix appearing in the selfenergy as

$$
\sum_{n} t_{m n}^{m^{\prime} n, \mathrm{pg}}\left(\zeta, q_{z} ; i \Omega\right)=\phi_{m m^{\prime}}^{2}(\zeta) t^{\mathrm{pg}}\left(\zeta, q_{z} ; i \Omega\right)
$$

with $t^{\mathrm{pg}}\left(\zeta, q_{z} ; i \Omega\right)$ defined in Eq. (17). Note this also justifies our definition of $\chi$ in Eq. (15).

In summary, beyond the weak attraction limit, pairs can form above the superconducting transition temperature. These pairs of electrons form in excited states rather than the quantum ground state. Interpreting the Gor'kov gap equation as a Bose Einstein condensation condition allows us to specify a precise ladder series structure for these non-condensed pairs. Because it will take us too far afield, elsewhere, we discuss a precise treatment of the parameter $\zeta$, in more microsopic detail ${ }^{5}$

\section{SPECIFYING THE PAIRING BASIS}

We now turn to the specific pairing choices that can be made to solve the system, and explore their physical effects. We now consider only a single $\zeta$, and for notational simplicity we now omit this parameter. Ensembles of $\zeta$ are discussed elsewhere ${ }^{[5}$ In order to make further progress, we must specify $\Delta(\boldsymbol{r})$ as well as the Landau level state basis we use. This requires that one determine the pairing partners of each fermionic Landau level state. Based on previous work ${ }^{6 / 9 \mid 10}$ here we analyze and compare two complementary choices for the case of an Abrikosov lattice. Throughout we use the Landau gauge $\boldsymbol{A}=(0, B \hat{\boldsymbol{x}}, 0)$ in which the energy gap of an Abrikosov lattice, $\Delta(\boldsymbol{r})$ is given by

$$
\begin{aligned}
\Delta(\boldsymbol{r}) & =C \sum_{m} \exp \left(i \pi \frac{b_{y}}{a} m^{2}\right) \psi_{0, m b_{x}, 0}^{\mathrm{cm}}(\boldsymbol{r}) \\
& =\Delta \sqrt{\frac{\sqrt{2} b_{x}}{L_{x} L_{y} L_{z} \sqrt{\pi} l_{H}}} \\
& \times \sum_{m} \exp \left[i \pi \frac{b_{y}}{a} m^{2}+\frac{2 i m b_{x} y}{l_{H}^{2}}-\frac{\left(x-m b_{x}\right)^{2}}{l_{H}^{2}}\right]
\end{aligned}
$$

where $\psi_{N, X, k_{z}}^{\mathrm{cm}}$ is the orbit-center Landau level state for a charge- $2 e$ particle with Landau level $N$, orbit center $X$, and $z$-momentum $k_{z}$. The normalization of $\Delta(\boldsymbol{r})$ is chosen such that $\int d \boldsymbol{r}|\Delta(\boldsymbol{r})|^{2}=|\Delta|^{2}$, with $L_{x}, L_{y}$, and $L_{z}$ the sample lengths and $l_{H}=\sqrt{\hbar c / e H}$ the Hall length. Finally, the Abrikosov lattice is characterized by unit vectors $\boldsymbol{a}=(0, a, 0)$ and $\boldsymbol{b}=\left(b_{x}, b_{y}, 0\right) . l_{H}$ is related to the unit vectors by $a b_{x}=\pi l_{H}^{2}$. For a square lattice $\boldsymbol{b}=$ $(a, 0,0)$ while for a triangular lattice $\boldsymbol{b}=(\sqrt{3} a / 2, a / 2,0)$, and in general we capture all Abrikosov lattices using the method in Ref. 13. $\zeta=b_{y} / a+i b_{x} / a$. Throughout the rest of this paper, we implicitly take $b_{x}, b_{y}$, and $a$ to be functions of $\zeta$ through the above formula and the restriction that $a b_{x}=\pi l_{H}^{2}$.

The two natural choices are to use either orbit-center wavefunctions, in which case orbit centers positioned symmetrically about a lattice site pair together, or to use magnetic translation group wavefunctions, in which case wavefunctions with opposite reciprocal lattice vectors are paired. We describe those pairs in terms of the notation of $\Delta_{m n}^{0}(\zeta)$ and $\phi_{m m^{\prime}}^{2}(\zeta)$ defined above.

\section{A. Orbit-center Pairing}

One choice of pairing, originally presented by Ryan and Rajagopal is to have fermions pair about lattice site positions in the orbit center basis.6|14 16 Because the bosonic wave functions forming the Abrikosov lattice are positioned at orbit centers $X=m b_{x}$, fermions which are equally spaced apart from these positions can pair, so that the pair wavefunction can be represented as $\Psi_{N_{1}, N_{2}, m b_{x}, Y, k_{z}}^{\text {pair }}(\boldsymbol{r})=$

$$
\psi_{N_{1}, m b_{x}+Y, k_{z}, \uparrow}^{\text {fermion }}(\boldsymbol{r}) \psi_{N_{2}, m b_{x}-Y,-k_{z}, \downarrow}^{\text {fermion }}(\boldsymbol{r}) .
$$

Here

$$
\begin{aligned}
& \psi_{N, X, k_{z}}^{\text {fermion }}(\boldsymbol{r})=\sqrt{\frac{1}{L_{y} L_{z} 2^{N} N !}}\left(\frac{1}{\pi l_{H}^{2}}\right)^{1 / 4} \\
& \times \exp \left(i k_{z} z+\frac{i X y}{l_{H}^{2}}-\frac{(x-X)^{2}}{2 l_{H}^{2}}\right) H_{N}\left(\frac{x-X}{l_{H}}\right)
\end{aligned}
$$

where $H_{N}$ is the $N$ th-order Hermite polynomial. The $\Delta_{m n}^{0}(\zeta)$ and associated factors are calculated in Appendix A. Quite generally (presuming inter-eigenstate pairs), the result is 
(a)

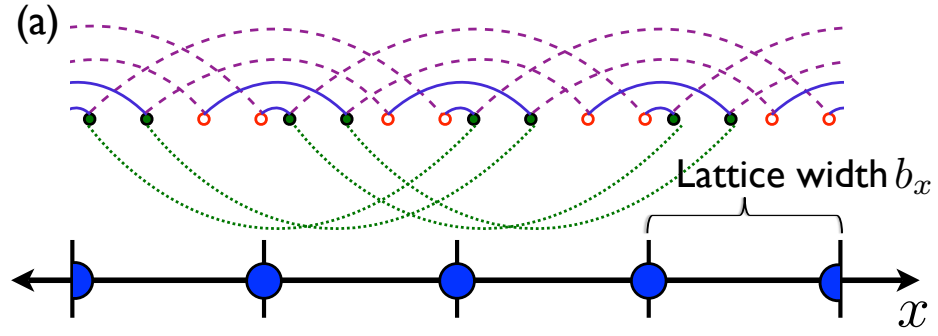

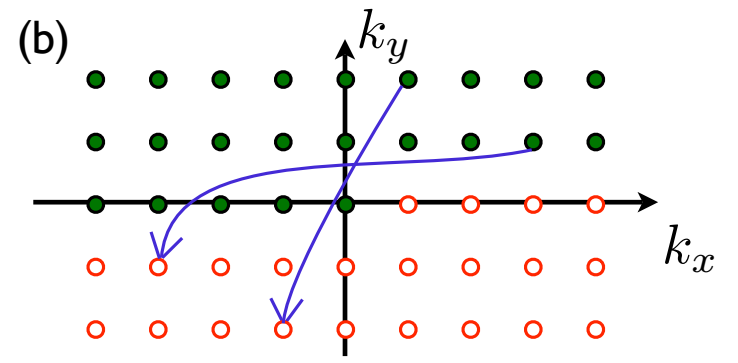

FIG. 2. (Color online) Schematic version of the two pairing models, (a) the restricted "tight-binding" pairing for case (i), in the case of a square lattice (see Section VII). The fermionic states (small open red and filled green circles) can pair with each other only via the solid blue (closest pairing partner) and dashed purple (next closest pairing partner) lines. These pairs in turn connect "nearest neighbor" states via an off-diagonal Green's function matrix element (dotted green lines; equivalent lines for the red states are omitted). Furthermore, the pairs can be interpreted as forming bosons at lattice sites (large blue circles). (b) The case (ii) version of pairing, in which states (filled green and open red circles) are in a reciprocal lattice of the magnetic translation group, and pair with states of opposite momentum index (blue lines).

$$
\Delta_{m=\left(N_{1}, X+Y, k_{z}\right), n=\left(N_{2}, X-Y,-k_{z}\right)}^{0}(\zeta)= \begin{cases}\sqrt{\frac{b_{x}}{L_{x} L_{y}^{2} L_{z}^{2} \pi l_{H}^{2}}} \frac{1}{2^{N_{1}+N_{2} \sqrt{N_{1} ! N_{2} !}}} e^{i \pi\left(b_{y} / a\right)\left(X / b_{x}\right)^{2}} e^{-Y^{2} / l_{H}^{2}} & \text { if } X=m b_{x}, m \in \mathbb{Z} \\ 0 & \text { otherwise. }\end{cases}
$$

\section{B. Magnetic Translation Group Pairing}

The other choice of pairing, originally presented by Dukan, Andreev, and Tesanovic ${ }^{7 / 10}$ and in parallel work by Akera, MacDonald, Norman, and Girvin, 17$][19$ and Nicopoulos and Kumar,20 is to use the magnetic translation group (MTG) for the fermions, with an index $\boldsymbol{k}{ }^{21}$ Following Ref. 10 we choose a unit lattice site for the magnetic translation group, which must be twice the size of the Abrikosov lattice site, with unit vectors $2 \boldsymbol{a}$ and $\boldsymbol{b}$. Then the reciprocal unit vectors are $\boldsymbol{a}^{*}=\left(-b_{y} / l_{H}^{2}, b_{x} / l_{H}^{2}\right)$ and $\boldsymbol{b}^{*}=\left(2 a / l_{H}^{2}, 0\right)$ so that $\boldsymbol{a}_{i} \boldsymbol{a}_{j}^{*}=2 \pi \delta_{i j}$ where $\boldsymbol{a}_{i}$ are the unit vectors and $\boldsymbol{a}_{j}^{*}$ are the reciprocal unit vectors. Restricting $\boldsymbol{k}=\left(k_{x}, k_{y}\right)$ to be within the limits of the cell $\left(\boldsymbol{a}^{*}, \boldsymbol{b}^{*}\right)$ gives a complete set of functions. ${ }^{22}$ We also note that $\psi$ is now dependent on $\zeta$, the configuration of the Abrikosov lattice.

This pairing occurs between opposite $\boldsymbol{k}$. To conform with our assumption of diagonal pairing, we need to specify that $N_{1}=N_{2}$, and then

$$
\Psi_{N, \boldsymbol{k}}^{\mathrm{pair}}(\boldsymbol{r})=\psi_{N, \boldsymbol{k}, k_{z}, \uparrow}^{\text {fermion }}(\boldsymbol{r}) \psi_{N,-\boldsymbol{k},-k_{z}, \downarrow}^{\text {fermion }}(\boldsymbol{r})
$$

with

$$
\begin{aligned}
\psi_{N, \boldsymbol{k}, k_{z}}^{\mathrm{fermion}} & =\sqrt{\frac{1}{2^{N} N !}}\left(\frac{1}{\pi l_{H}^{2}}\right)^{1 / 4} \sqrt{\frac{b_{x}}{L_{x} L_{y} L_{z}}} \exp \left(i k_{z} z\right) \sum_{m} \exp \left(i \frac{\pi b_{y}}{2 a} m^{2}+i m k_{x} b_{x}\right) \\
& \times \exp \left\{i\left(k_{y}+\frac{\pi m}{a}\right) y-\frac{\left[x-\left(k_{y}+\frac{\pi m}{a}\right) l_{H}^{2}\right]}{2 l_{H}^{2}}\right\} H_{n}\left\{\left[x-\left(k_{y}+\frac{\pi m}{a}\right) l_{H}^{2}\right] / l_{H}\right\} .
\end{aligned}
$$

The $\Delta_{m n}^{0}(\zeta)$ and associated factors are calculated in Refs. 9] and 10, with the result that for the lowest Landau level

$$
\begin{aligned}
& \Delta_{m=\left(0, \boldsymbol{k}, k_{z}\right), n=\left(0,-\boldsymbol{k},-k_{z}\right)}^{0}(\zeta)=\sqrt{\frac{b_{x}}{L_{x} L_{y} L_{z} \sqrt{2 \pi} l_{H}}} \\
& \quad \times \exp \left(-\left(k_{y} l_{H}\right)^{2}\right) \theta_{3}\left(\left[-k_{x}+i k_{y}\right] b_{x} \mid \frac{-b_{y}}{a}+\frac{i \pi l_{H}^{2}}{a^{2}}\right),
\end{aligned}
$$


where $\theta_{3}(u \mid \tau)=\sum_{n=-\infty}^{\infty} \exp \left(2 i n u+i \pi n^{2} \tau\right)$ is the third elliptic theta or Jacobi theta function. Further $\Delta_{m n}^{0}(\zeta)$ for higher Landau levels can be iteratively calculated from this 10

\section{NEAREST NEIGHBOR PAIRING APPROXIMATION FOR REAL SPACE PAIRING}

While the MTG method for pairing results in each fermion pairing with exactly one other degenerate eigenstate, that is not the case for orbit center pairing. If $X$ is a lattice site, then a fermion at $X+Y$ can pair not only with $X-Y$, but also with $X-Y+2 n b_{x}$ with $n \in \mathbb{Z}$. A very complicated matrix thus results for the Green's function, but here we demonstrate that this matrix can be substantially simplified such that it is analytically tractable yet incorporates the important physics.

We begin by noting that $\Delta_{m n}^{0}$ is proportional to $\exp \left(-Y^{2} / l_{H}^{2}\right)$ where $Y$ is half of the distance between the two states that pair. Thus, pairing between states that are well separated will be exponentially suppressed. Using only a single pairing partner is inadequate, however, because it fails to capture interference effects between two pairing partners, as will be demonstrated below. Using two pairing partners does capture these effects and reproduces the correct local density of states for the lattice. This is reminiscent of a hierarchical "tightbinding" scheme, in that the nearest pairing partner can be considered an "on-site" interaction, and the secondnearest pairing partner allows for interactions between lattice sites.

To implement this approach, we begin with Eq. 7 , $G_{m m^{\prime}}(i \omega)=$

$$
G_{m}^{0}(i \omega) \delta_{m m^{\prime}}-G_{m}^{0}(i \omega) \sum_{l n} \Delta_{m l} G_{l}^{0}(-i \omega) \Delta_{l n}^{\dagger} G_{n m^{\prime}}(i \omega)
$$

This can be further simplified by separately including the Green's functions of pairing partners, resulting in a new equation for the Green's functions $G_{m m^{\prime}}(i \omega)=$

$$
G_{m}^{0}(i \omega) \delta_{m n}+G_{m}^{0}(i \omega) \sum_{n} \Delta_{m n}\left[-\tilde{G}_{n m^{\prime}}(i \omega)\right]
$$

Here by $\tilde{G}$ we mean (a) flipping the sign of $i \omega$ and (b) conjugating the $\Delta$ appearing within $G$. By this definition, we also have that $\tilde{G}_{m m^{\prime}}(i \omega)=$

$$
G_{m}^{0}(-i \omega) \delta_{m m^{\prime}}+G_{m}^{0}(-i \omega) \sum_{n} \Delta_{m n}^{\dagger} G_{n m^{\prime}}(i \omega)
$$

This correctly reproduces the original Green's function above, and by inverting Eq. (34), we have that

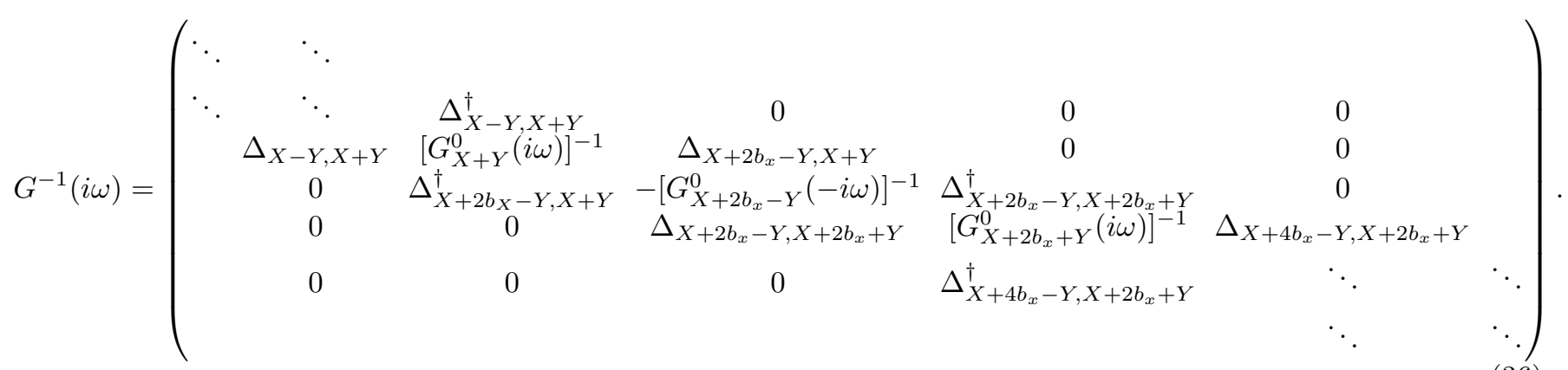

Here we use only the index corresponding to the orbit center, which is the only one which varies throughout this matrix. We also take $Y>0$; if $Y<0$ positions in the matrix will flip but it will be otherwise unchanged. Also note that the values of $G_{0}^{-1}$ are actually independent of orbit center; the indices remain for clarity.

This Green's function matrix is computationally simplified as compared to the full orbit-center pairing scheme. Furthermore, in the case of a square or triangular lattice, its eigenvalues can be found analytically. For both a square lattice $(\zeta=i)$ and a triangular lattice $(\zeta=1 / 2+\sqrt{3} i / 2)$, we have the property that $\Delta_{X-Y+2 b_{x}, X+Y+2 b_{x}}^{0}=\Delta_{X-Y, X+Y}^{0}$, implying that the entries in $G^{-1}$ repeat with period two along the diagonal. We can then posit a plane-wave solution for the eigen- vectors, with $a$ and $b$ constants:

$$
\boldsymbol{v}=\sum_{q} e^{i q k_{j}}\left(a\left|X_{\bar{m}}+2 q b_{x}\right\rangle+b\left|X_{m}+2 q b_{x}\right\rangle\right),
$$

where for compactness we set $\bar{m}=X-Y, m=X+Y$, and $\bar{n}=X-Y+2 b_{x}$, using the symmetry properties of $\Delta_{m n}^{0}$. We obtain

$$
G^{-1} \boldsymbol{v}=\left(\begin{array}{c}
\vdots \\
\Delta_{\bar{m} m} b+(\omega-\xi) a+\Delta_{\bar{n} m} e^{i k_{j} b} \\
\Delta_{\bar{n} m}^{\dagger} a+(\omega+\xi) e^{i k_{j}} b+\Delta_{\bar{m} m}^{\dagger} e^{i k_{j}} a \\
\vdots
\end{array}\right)
$$

The energy eigenvalues of this system will be zero eigenvalues of $G^{-1}$. For a nontrivial solution, 


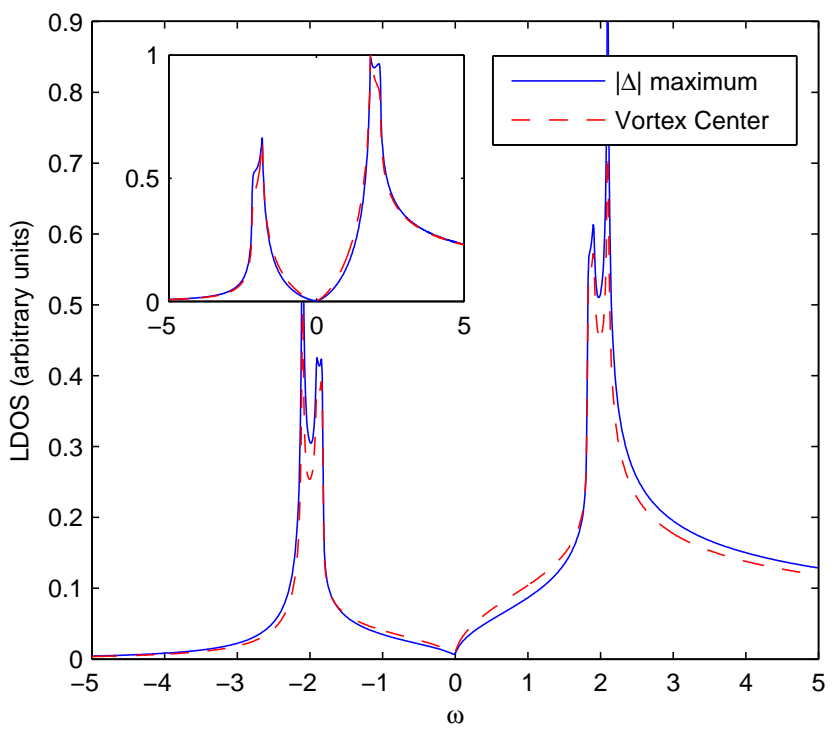

FIG. 3. (Color online) Plots of the local density of states $N(\boldsymbol{r} ; \omega)$ vs. $\omega$ on a square lattice, for $\Delta^{\mathrm{sc}}=2$ and $\delta=0.01$, and normalized to a maximum LDOS of 1.0. (Main figure) Case (i), using the nearest neighbor approach for calculations with $L_{x}=4 b_{x}$. (Inset) Case (ii).

we obtain $-\Delta_{\bar{n} m}^{\dagger}\left(\Delta_{\bar{m} m} e^{-i k_{j}}+\Delta_{\bar{n} m}\right)+\left(-\omega^{2}-\xi^{2}\right)-$ $\Delta_{\bar{m} m}^{\dagger}\left(\Delta_{\bar{m} m}+\Delta_{\bar{n} m} e^{i k_{j}}\right)=0$. We thus have $-\omega^{2}=$

$\xi^{2}+\left|\Delta_{\bar{m} m}\right|^{2}+\left|\Delta_{\bar{n} m}\right|^{2}+e^{-i k_{j}} \Delta_{\bar{m} m} \Delta_{\bar{n} m}^{\dagger}+e^{i k_{j}} \Delta_{\bar{m} m}^{\dagger} \Delta_{\bar{n} m}$.

Here $\Delta_{\bar{m} m}=C_{\bar{m} m} e^{i \pi\left(b_{y} / a\right)\left(X / b_{x}\right)^{2}}$, where $C_{\bar{m} m}$ is a real number, and $\Delta_{\bar{n} m}=C_{\bar{n} m} e^{i \pi\left(b_{y} / a\right)\left[(X+1) / b_{x}\right]^{2}}$. We further simplify

$$
\begin{aligned}
& e^{-i k_{j}} \Delta_{\bar{m} m} \Delta_{\bar{n} m}^{\dagger}+e^{i k_{j}} \Delta_{\bar{m} m}^{\dagger} \Delta_{\bar{n} m} \\
& =2 \operatorname{Re}\left(e^{-i k_{j}} \Delta_{\bar{m} m} \Delta_{\bar{n} m}^{\dagger}\right) \\
& =2 C_{\bar{m} m} C_{\bar{n} m} \operatorname{Re}\left(e^{-i k_{j}} e^{ \pm i \pi\left(b_{y} / a\right)}\right)
\end{aligned}
$$

where we have used the $b_{x}$ and $b_{y}$ specific to a square or triangular lattice, and the + is for $X / b_{x}$ an odd integer, - for $X / b_{x}$ an even integer. We then have $-\omega^{2}=$

$\xi^{2}+\left|\Delta_{\bar{m} m}\right|^{2}+\left|\Delta_{\bar{n} m}\right|^{2}+2 C_{\bar{m} m} C_{\bar{n} m} \operatorname{Re}\left(e^{-i k_{j}} e^{ \pm i \pi\left(b_{y} / a\right)}\right)$.

We also note that for a system of $L_{x}=2 N_{x} b_{x}$, we must have $N_{x} k_{j}=2 \pi j$ to enforce periodic boundary conditions, giving $k_{j}=2 \pi j / N_{x}$ with $j$ ranging from 0 to $N_{x}-1$. With this approximation in place, we may turn to the results of these pairing theories.

\section{RESULTS AND DISCUSSION}

This paper has been rather extensively devoted to theoretical formalism which characterizes the fermionic degrees of freedom. Among the most direct experimentally

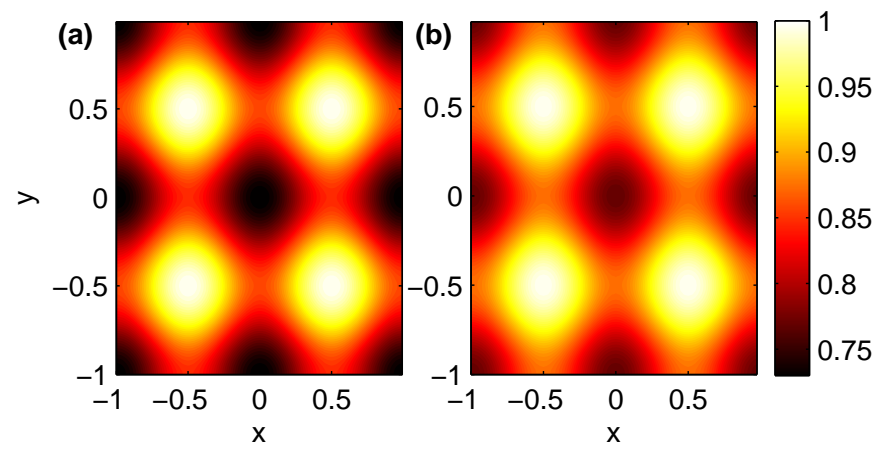

FIG. 4. (Color online) Plots of the local density of states $N(\boldsymbol{r} ; \omega)$ for (a) case (i) and (b) case (ii), normalized to the local density of states at the vortex cores (located at $(x, y)=$ $( \pm 0.5, \pm 0.5))$ and as a function of position on a square lattice, for $\omega=0.4, \Delta^{\mathrm{sc}}=2$, and $\delta=0.05$ (slightly higher than Fig. 3 to permit faster computation without a substantial change in accuracy).

relevant consequences is the behavior of the local density of states (LDOS) $N(\boldsymbol{r} ; \omega)$ vs. $\omega$. Here we address this density of states in the very low temperature regime. This is experimentally accessible using scanning tunneling microscopy ${ }^{23}$ The local density of states $N(\boldsymbol{r} ; \omega)$ is calculated via $N(\boldsymbol{r} ; \omega)=2 \operatorname{Im} G^{\mathrm{ret}}(\boldsymbol{r}, \boldsymbol{r} ; \omega)$. We determine $G^{\mathrm{ret}}\left(\boldsymbol{r}, \boldsymbol{r}^{\prime} ; \omega\right)=\sum_{m m^{\prime}} G_{m m^{\prime}}(\omega+i \delta) \psi_{m}(\boldsymbol{r}) \psi_{m^{\prime}}^{\dagger}\left(\boldsymbol{r}^{\prime}\right)$ in the limit $\delta \rightarrow 0^{+}$, and for simplicity we address the square lattice at the lowest temperature where there is only a condensate. Also for simplicity our illustrative calculations are for the limiting case of the lowest Landau level, an $s$-wave gap, $N_{x}=2$ for orbit center pairing, and normalized to set the mass $m=1$.

It has been argued quite generally that $N(\boldsymbol{r} ; \omega)$ exhibits a gapless behavior, in contrast to superconductivity without a magnetic field. This gaplessness is $\mathrm{du} \mathrm{e}^{[1]}$ to the fact that all fermions are delocalized, unlike in the vortex cores of the low field limit. This observation has direct application to magnetic oscillation measurements, as a gapped state would dampen these oscillations significantly! ${ }^{1}$ More specifically in the MTG pairing scheme, systematic studies in Ref. [10 show that the energy eigenvalues satisfy $E_{N, \boldsymbol{k}, k_{z}}=\xi_{N, k_{z}}^{2}+$ $\left|\Delta_{m=\left(N, \boldsymbol{k}, k_{z}\right), n=\left(N,-\boldsymbol{k},-k_{z}\right)}\right|^{2}$. Because $\Delta_{m n}$ always features a zero for the Abrikosov lattice, $E$ will as well.

The situation for orbit center pairing is more complex, but with the nearest neighbor pairing scheme above, we are able to analytically demonstrate gaplessness in this case as well as for the square and triangular Abrikosov lattices. Such gaplessness will occur when $Y=0.5 b_{x}$, which means the magnitudes of $\left|\Delta_{\bar{m} m}\right|^{2}+\left|\Delta_{\bar{n} m}\right|^{2}$ and $2\left|\Delta_{\bar{n} m} \Delta_{\bar{m} m}^{\dagger}\right|$ are equal (using the notation from the previous section). Thus, for gaplessness to occur, we must also have that

$$
\arg \left(e^{-i k_{j}} e^{ \pm i \pi\left(b_{y} / a\right)}\right)=\pi
$$


or $j_{\text {gapless }}=\frac{N_{x}}{2}\left(1 \pm \frac{b_{y}}{a}\right.$. $)$ For a square lattice, any $N_{x}=2 m, m \in \mathbb{N}$ will thus feature a gapless state, while for a triangular lattice, any $N_{x}=4 m$ will feature a gapless state. This also shows the importance of including "nearest-neighbor" pairs; neglecting them cannot produce gapless states.

Beyond this analytical assessment, we observe additional similarities and differences between the pairing schemes, and gain intuition about experiment. Figure 3 presents a plot of the local density of states as a function of energy for the two different cases and for two different positions of the probe: one at the vortex center (dashed) and the other at the point of maximum $|\Delta|$ (solid curve). Again prominent in the features of these LDOS plots is that both cases show gapless behavior. In both cases, there is only one "nodal" state $m$ per lattice site at which its total excitation $E=0$. This produces the distinctive parabola-like shape in Fig. 3, which still touches $N(\boldsymbol{r} ; 0)=0$ but does not exhibit a complete gap away from $\omega=0$. The variation between the solid and dashed curves is rather small, also reflecting this point.

Figure 4 presents a contour plot of $N(\boldsymbol{r} ; \omega)$ as a function of $\boldsymbol{r}$. This figure nicely illustrates that the real space and reciprocal lattice space pairing schemes are rather similar here. Both reflect the symmetry of the Abrikosov lattice. However the former shows slightly more contrast than the latter. 24

\section{CONCLUSIONS}

It is anticipated that the formalism in this paper will be relevant to both ultracold gases in the BCS-BEC crossover regime, and possibly to the pseudogap phase in high temperature superconductors. Magnetic field effects in the latter have revealed a number of mysteries, which appear to be associated with a normal state pairing gap. For the cold Fermi gases there is considerable interest in effects arising from artificial gauge fields or rapid rotation. Previous work on these Fermi gases ${ }^{25}$ has presumed, rather improbably, that even in the BEC regime, pairing and condensation appear at the same temperature. Also important is a better understanding of the normal state and of how condensation can take place if the superconductor or superfluid is effectively one dimensional.

This paper has focused on the nonlinear gap region in the presence of a magnetic field. A next step is to address calculations of the onset of superfluid coher- ence at temperature $T_{c}(H)$, which is taken to be less than the onset of pairing, $T^{*}(H)$, in contrast to previous work. $\frac{36 / 7|10| 14|25| 26}{16}$ To this end, our work has established that the Gor'kov equations lend themselves to the nonlinear, analytic approach required, provided only degenerate energy states are paired. It has also shown that unique pairing partners are not required for a tractable theory. A robust result of this theory is that gapless states are present for both real and momentum-space pairing theories in a very high field.

In summary, it is hoped that this formalism lays the foundation to explore a variety of magnetic field effects in the pseudogap phase and throughout the BCS-BEC crossover.

We thank Victor Gurarie, Tin-Lun Ho, and Vivek Mishra for helpful discussions. This work is supported by NSF-MRSEC Grant 0820054. P.S. acknowledges support from the Hertz Foundation.

\section{Appendix A: Details of the Orbit-Center Pairing Calculations}

Since $\Psi^{\text {pair }}$ is independent of $k_{z}$, that subscript will be dropped in pair terms that follow.

Ref. 6 provides an identity,

$$
\begin{aligned}
& \psi_{N_{1}, X+Y}\left(\boldsymbol{r}_{1}\right) \psi_{N_{2}, X-Y}\left(\boldsymbol{r}_{2}\right)=\sum_{P=0}^{N_{1}+N_{2}} C_{N_{1} N_{2}}^{P} \\
& \times \psi_{P, X}^{\mathrm{cm}}\left(\boldsymbol{r}_{\mathrm{cm}}\right) \psi_{N_{1}+N_{2}-P, 2 Y}^{\mathrm{rel}}\left(\boldsymbol{r}_{\mathrm{rel}}\right)
\end{aligned}
$$

where $N_{1}, N_{2}$, and $P$ are Landau levels, $\boldsymbol{r}_{c m}=\left(\boldsymbol{r}_{1}+\right.$ $\left.\boldsymbol{r}_{2}\right) / 2, \boldsymbol{r}_{\mathrm{rel}}=\boldsymbol{r}_{1}-\boldsymbol{r}_{2}, \psi^{\mathrm{cm}}$ is an orbital function with $l \rightarrow 2^{-1 / 2} l$ (appropriate for a charge-2e particle), $\psi^{\text {rel }}$ has $l \rightarrow 2^{1 / 2} l$, and $C_{N_{1} N_{2}}^{P}$ is a complicated combinatorial prefactor which is equal to 1 if $N_{1}=N_{2}=0$. Thus, we know that

$$
\begin{aligned}
& \Psi_{N_{1}, N_{2}, X, Y}^{\mathrm{pair}}(\boldsymbol{r})=\sum_{P=0}^{N_{1}+N_{2}} C_{N_{1} N_{2}}^{P} \psi_{P, X}^{\mathrm{cm}}(\boldsymbol{r}) \psi_{N_{1}+N_{2}-P, 2 Y}^{\mathrm{rel}}(0) \\
& =\sum_{P=0}^{N_{1}+N_{2}} C_{N_{1} N_{2}}^{P} \sqrt{\frac{1}{L_{y} L_{z} 2^{N_{1}+N_{2}-P}\left(N_{1}+N_{2}-P\right) !}} \\
& \times\left(\frac{1}{2 \pi l_{H}^{2}}\right)^{1 / 4} e^{-Y^{2} / l_{H}^{2}} \psi_{P, X}^{\mathrm{cm}}(\boldsymbol{r})
\end{aligned}
$$

We can now proceed to calculate the $\Delta_{m n}$ elements for all possible pairs, with the result that 


$$
\begin{aligned}
& \Delta_{m=\left(N_{1}, X+Y\right), n=\left(N_{2}, X-Y\right)}=\int d \boldsymbol{r} \Delta(\boldsymbol{r}) \psi_{N_{1}, X+Y, k_{z}}^{\text {fermion } \dagger}(\boldsymbol{r}) \psi_{N_{2}, X-Y,-k_{z}}^{\text {fermion } \dagger}(\boldsymbol{r}) \\
& =\Delta \sqrt{\frac{\sqrt{2} b_{x}}{L_{x} L_{y} L_{z} \sqrt{\pi} l_{H}}} \int d \boldsymbol{r} \sum_{m} \exp \left(i \pi \frac{b_{y}}{a} m^{2}\right) \psi_{0, m b_{x}}^{\mathrm{cm}}(\boldsymbol{r}) \\
& \times \sum_{P=0}^{N_{1}+N_{2}} C_{N_{1} N_{2}}^{P} \sqrt{\frac{1}{L_{y} L_{z} 2^{N_{1}+N_{2}-P\left(N_{1}+N_{2}-P\right) !}}\left(\frac{1}{2 \pi l_{H}^{2}}\right)^{1 / 4} e^{-Y^{2} / l_{H}^{2}} \psi_{P, X}^{\mathrm{cm} \dagger}(\boldsymbol{r})} \\
& = \begin{cases}\Delta \sqrt{\frac{\sqrt{2} b_{x}}{L_{x} L_{y} L_{z} \sqrt{\pi} l_{H}}} e^{i \pi\left(b_{y} / a\right)\left(X / b_{x}\right)^{2}} C_{N_{1} N_{2}}^{0} \sqrt{\frac{1}{L_{y} L_{z} 2^{N_{1}+N_{2}\left(N_{1}+N_{2}\right) !}}}\left(\frac{1}{2 \pi l_{H}^{2}}\right)^{1 / 4} e^{-Y^{2} / l_{H}^{2}} & \text { if } X=m b_{x}, m \in \mathbb{Z}, \\
0 & \text { otherwise. }\end{cases}
\end{aligned}
$$

Here $C_{N_{1} N_{2}}^{0}=\sqrt{\frac{\left(N_{1}+N_{2}\right) !}{N_{1} ! N_{2} !}} \frac{(-1)^{N_{2}}}{2^{\left(N_{1}+N_{2}\right) / 2}}$ to give

$$
\Delta_{m=\left(N_{1}, X+Y\right), n=\left(N_{2}, X-Y\right)}= \begin{cases}\Delta \sqrt{\frac{b_{x}}{L_{x} L_{y}^{2} L_{z}^{2} \pi l_{H}^{2}}} \frac{1}{2^{N_{1}+N_{2}} \sqrt{N_{1} ! N_{2} !}} e^{i \pi\left(b_{y} / a\right)\left(X / b_{x}\right)^{2}} e^{-Y^{2} / l_{H}^{2}} & \text { if } X=m b_{x}, m \in \mathbb{Z} \\ 0 & \text { otherwise }\end{cases}
$$

1 T. Maniv, V. Zhuravlev, I. Vagner, and P. Wyder, Rev. Mod. Phys. 73, 867 (2001)

${ }^{2}$ N. Doiron-Leyraud, C. Proust, D. LeBoeuf, J. Levallois, J. B. Bonnemaison, R. Liang, D. A. Bonn, W. Hardy, and L. Taillefer, Nature 447, 565 (2007).

${ }^{3}$ M. Rasolt and Z. Tesanovic, Rev. Mod. Phys. 64, 709 (1992)

${ }^{4}$ P. A. Lee and S. R. Shenoy, Phys. Rev. Lett. 28, 1025 (1972)

P. Scherpelz, D. Wulin, K. Levin, and A. K. Rajagopal, arXiv:1112.1112v2 (2012).

6 J. C. Ryan and A. K. Rajagopal, Phys. Rev. B 47, 8843 (1993)

S. Dukan, A. V. Andreev, and Z. Tesanovic, Physica C 183, 355 (1991)

${ }^{\circ}$ Z. Tesanovic and P. D. Sacramento, Phys. Rev. Lett. 80, 1521 (1998).

${ }^{9}$ M. G. Vavilov and V. P. Mineev, J. Exp. Theor. Phys. 85, 1024 (1997).

Tó S. Dukan and Z. Tesanovic, Phys. Rev. B 49, 13017 (1994).

11 Q. Chen, J. Stajic, S. Tan, and K. Levin, Physics Reports 412, 1 (2005).

${ }^{12}$ A. H. MacDonald, H. Akera, and M. R. Norman, Phys. Rev. B 45, 10147 (1992).

Is D. Saint-James, G. Sarma, and E. J. Thomas, Type II superconductivity (Pergamon Press, Oxford, 1969).

14 A. K. Rajagopal, Phys. Rev. B 46, 1224 (1992).

15 A. K. Rajagopal, in Selected topics in superconductivity, edited by L. C. Gupta and M. S. Multani (World Scientific, 1993) pp. 193-209.

16 A. K. Rajagopal and R. Vasudevan, in Selected Topics in Mathematical Physics: Professor R. Vasudevan Memorial Volume, edited by R. Sridhar, K. Srinivasa Rao, and V. Lakshminaryanan (Allied Publishers Ltd, New Delhi, 1995) pp. 129-165.

17 H. Akera, A. H. MacDonald, S. M. Girvin, and M. R. Norman, Phys. Rev. Lett. 67, 2375 (1991)

18 M. Norman, H. Akera, and A. MacDonald, Physica C: Superconductivity 196, 43 (1992)

${ }_{19}$ M. R. Norman, A. H. MacDonald, and H. Akera, Phys. Rev. B 51, 5927 (1995)

${ }^{20}$ V. N. Nicopoulos and P. Kumar, Phys. Rev. B 44, 12080 (1991)

${ }^{21}$ Note that Refs. 7 and 10 use a different gauge than we use here.

22 Y. A. Bychkov and E. I. Rashba, Sov. Phys. JETP 58, 1062 (1983).

23 O. Fischer, M. Kugler, I. Maggio-Aprile, C. Berthod, and C. Renner, Rev. Mod. Phys. 79, 353 (2007)

24 Topographic plots at higher frequencies (not shown) have slight deviations from an Abrikosov lattice symmetry for case (i), due to the small $N_{x}=2$. Similarly, the "kinks" in the low-frequency LDOS are likely due to the same approximation.

25 H. Zhai and T.-L. Ho, Phys. Rev. Lett. 97, 180414 (2006); M. Y. Veillette, D. E. Sheehy, L. Radzihovsky, and V. Gurarie, ibid. 97, 250401 (2006); G. Moller and N. R. Cooper, ibid. 99, 190409 (2007).

26 A. K. Rajagopal and J. C. Ryan, Phys. Rev. B 44, 10280 (1991) 